\title{
A posterior epidural mass causing paraparesis in a 20-year-old healthy individual
}

\author{
Tarkan Ergun • Hatice Lakadamyali • Ertan Gokay
}

Received: 28 February 2008 / Accepted: 13 June 2009 / Published online: 26 August 2009

(C) Springer-Verlag London Ltd 2009

\begin{abstract}
We present a case of a posterior epidural abscess at the thoracic vertebral level causing paraparesia in a young, healthy individual with no otherwise predisposing factors, with normal laboratory findings, as diagnosed using fat-suppressed MR imaging. Spinal epidural abscess is a rare condition, encountered mostly in the midthoracic or lower lumbar vertebral levels of elderly patients, that has a high mortality and morbidity (18-31\%) when diagnosis and treatment is delayed. It is rarely spontaneous and is usually accompanied by spinal osteomyelitis. Diagnosis is rather easy in cases of vertebral osteomyelitis or when classical clinical, laboratory and imaging findings are present. However, cases of spontaneous development, with no predisposing factors, and lack of abscess suggesting clinical and laboratory findings may be a diagnostic challenge. In such cases, other posterior epidural masses such as schwannoma, neurofibroma, meningioma and hematoma should be considered in the differential diagnosis. Both the clinician and the radiology physician should have a high suspicion of epidural abscesses, because their early
\end{abstract}

\footnotetext{
T. Ergun $\cdot$ H. Lakadamyali

Department of Radiology, Baskent University,

Alanya Teaching and Medical Research Center,

Alanya, Turkey

E. Gokay

Department of Family Medicine, Baskent University,

Alanya Teaching and Medical Research Center,

Alanya, Turkey

T. Ergun $(\bowtie)$

Department of Radiology,

Baskent University School of Medicine,

Alanya Research and Training Hospital,

07400 Alanya, Antalya, Turkey

e-mail: tarkanergun@yahoo.com
}

diagnosis and treatment is important. In addition to routine MR images, fat-suppressed MR images prove helpful in the diagnosis of spontaneous epidural abscesses by showing the inflammatory changes in the paraspinal area.

Keywords Posterior epidural mass $\cdot$ MRI $\cdot$ Spontaneous epidural abscesses

\section{Introduction}

Spinal epidural abscess (SEA) is a rare suppurative pathology generally accompanied by spinal osteomyelitis [1]. The reported incidence ranges from $0.2-1.2$ in 10,000 hospital presentations [2]. It most frequently occurs following lumbar interventions, epidural anesthesia and spinal surgery. However, spontaneous spinal epidural abscess is a very rare entity that is typically seen in immunocompromised patients (diabetes, cirrhosis, AIDS, long-term steroid use, malignancies and chronic renal failure dialysis cases) secondary to dissemination of systemic or local infections [3]. SEA is a life-threatening condition, and its early diagnosis and therapy are critical to prevent mortality and serious morbidity.

Here we present a case of a spontaneous posterior epidural abscess occuring in a young, healthy patient without predisposing risk factors, laboratory or radiographic abnormalities.

\section{Case presentation}

A 20-year-old male presented to the emergency department complaining of neck pain for the last 3 weeks and 
numbness in his lower extremities for the last 2 days. His past medical history was unremarkable. He denied any recent trauma. His blood pressure was $125 / 80 \mathrm{mmHg}$, heart rate was 88 beats $/ \mathrm{min}$, breathing was 16 breaths $/ \mathrm{min}$, and body temperature was $36.7^{\circ} \mathrm{C}$. Physical examination revealed hyperactive deep tendon reflexes in the lower extremities. Motor power examination revealed a $4 / 5$ score for both lower extremities. There was no skin infection in the cervical area. All laboratory tests were within normal limits [white blood cell count of $7.4 \times 109 / 1$, erythrocyte sedimentation rate (ESR) of $17 \mathrm{~mm} / \mathrm{h}$ and a C-reactive protein (CRP) of $8 \mathrm{mg} / \mathrm{l}$. The cervical X-ray was normal. A thoracic MRI was obtained and revealed a mass in the T2-6 posterior epidural space compressing the spinal cord and causing myelopathy. The neighboring bony structures were intact. The epidural mass demonstrated significant contrast enhancement following intravenous gadolinium administration. The fat-suppression images revealed edema and inflammatory changes in the posterior paraspinal area in the mass vicinity (Fig. 1), and the patient was scheduled for surgery with the diagnosis of posterior epidural abscess.

The surgical intervention was accomplished using a posterior approach technique. Partial laminectomy was done, granulation tissue and yellow purulent collection were drained. Staphylococcus aureus was cultured in the microbiology laboratory. A 3-week intravenous therapy followed by 6 weeks of oral antistaphylococcal therapy was instituted. The motor functions improved fully in the 4 th week. The control visit 1 year later revealed no abnormal clinical and neurological findings.

\section{Discussion}

The classical clinical triad of findings in SEA is back pain, progressive neurological deficit and fever [1]. This classical triad, however, is observed in only a small portion of the cases (37\%). The most frequent early clinical findings are fever (67\%) and spinal ache (89\%). Neurological deficits appear with the extension of the infection to the epidural space. The clinical course of SEA goes through the following successive stages: spinal ache, radicular pain, weakness and paralysis. However, the time elapsing between onset of symptoms and appearance of neurological deficits may vary from hours to months.

Spinal ache is the most frequent symptom and is present, as was the case with our patient, in almost all patients and all through the course of the disease. The localized spinal ache is persistent and is not relieved with bed rest. It has been designated as the most characteristic symptom of SEA in all studies.
It is, however, non-specific and, regrettably, does not indicate a serious medical condition. It is hard to diagnose SEA early because of its rarity and because of the nonspecific nature of its symptoms. The lack of related sensitive and specific laboratory tests adds further to the difficulty of early diagnosis. The ratio of correct initial diagnoses of SEA was reported to be $11 \%$ in the study of Tang et al. and $17 \%$ in the study of Lu et al. [4, 5]. Most of the clinical findings in SEA overlap with symptoms and findings of other pathological conditions. Other infections affecting the spinal cord and its neighboring structures (especially tuberculosis) predominate in the diagnosis whenever fever, spinal tenderness and spinal ache are present. As with our patient, in cases where back pain and paralysis of the lower extremities are present, disc and vertebral degenerative diseases, primary or secondary tumors, and fractures predominate in the diagnosis. In cauda equina syndrome cases, sphincter deficit and sensory deficits are present in addition to motor deficits of the lower extremities.

Contrary to our case, spinal epidural abscess is encountered in patients between $30-70$ years of age and in the lower thoracic and lumbar regions, and is usually accompanied by osteomyelitis [6]. Spontaneous cases are quite rare. In nearly $80 \%$ of SEA cases risk factors such as diabetes, cancer, degenerative spinal disease, tumors, AIDS, intravenous drug abuse, alcoholism and steroid intake are present. The most frequently observed risk factor is diabetes mellitus, being present in $18-54 \%$ of cases. Evaluation for risk factors is extremely important with regard to diagnosis. One study suggests that risk factor assessment offers a better sensitivity than the traditional triad of SEA, without significant loss of specificity [5].

Spinal epidural abscess may develop as a spread from a neighboring osteomyelitis focus or as a hematogenous spread from distant sites (usually skin or subcutaneous tissue) [7]. Ventral abscesses are rare, and usually the broad dorsal extradural area, which is rich in venous supply, is involved.

When a spinal epidural abscess is suspected, leukocyte count, erythrocyte sedimentation rate and C-reactive protein may be helpful in showing the infection. The inflammation indicators ESR and CRP are quite sensitive, but have low specificity. ESR is a sensitive laboratory indicator for pyogenic infections and is positive in more than $90 \%$ of cases. The mean ESR value in SEA patients is $51 \mathrm{~mm} / \mathrm{h}$. Serum CRP, on the other hand, is elevated within $6 \mathrm{~h}$ of a bacterial infection and is seen in more than $90 \%$ of cases, being more specific than ESR. It is interesting to note, however, that the ESR and CRP values, which would normally guide the diagnostic workup, were within normal limits in our patient. The white blood cell count is normal in half of the SEA cases, and if elevation is 

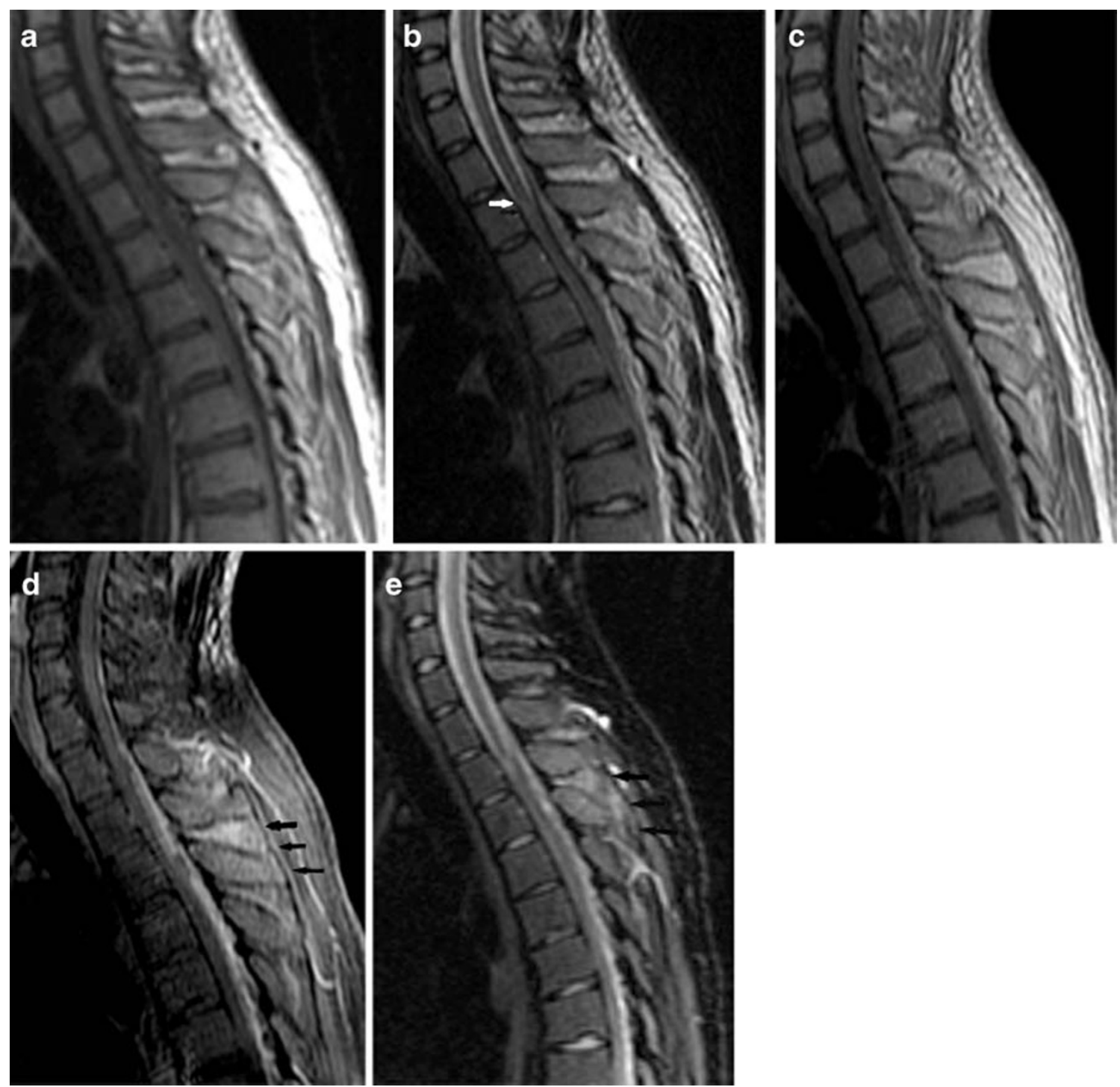

Fig. 1 Sagittal thoracic MRI images demonstrating a T2-6 posterior epidural mass lesion, hypointense in the T1-weighted (a) and hyperintense in the $\mathrm{T} 2$-weighted sequences (b), demonstrating significant contrast attenuation (except for a small necrotic area) following intravenous gadolinium application (c). The mass is noticed to compress the spinal cord and cause myelopathy (white arrow). No

present, it is usually a moderate one and does not contribute to the SEA diagnosis. Blood cultures may grow the infecting organism, especially in those with hematogenous spread and in intravenous drug abusers [8]. The most frequently grown organism in cultures is Staphylococcus aureus [9].

Lumbar puncture is not indicated as it carries the risk of spreading the infective agent into the subarachnoid space and thus may cause meningitis.

Direct roentgenograms provide useful information in only $20 \%$ of cases. Despite being noncontributory to the SEA diagnosis, it can demonstrate accompanying osteomyelitis and fracture. Narrowing of intervertebral disc pathological signal changes are noted in the bony structures and paraspinal area; fat-suppressed T2-weighted (d) and T1-weighted fatsuppressed sequences following intravenous gadolinium injection (e) demonstrating edema and inflammatory changes of the paraspinal area in addition to the extradural mass (black arrows)

space is noticed on direct roentgenograms 2-3 weeks after the onset of the infection. Because a large number of patients have degenerative changes on imaging studies, changes caused by infection on top of degenerative changes are not so apparent in the early stage of infectious disease. Therefore, a high level of suspicion must be maintained when evaluating radiographs for spinal infection. After 8 to 12 weeks, endplate sclerosis can be seen from reactive bone formation.

The most effective visualization modality of spinal epidural abscess is MRI. The epidural mass demonstrates homogeneous contrast enhancement in the early course of the disease and peripheral contrast enhancement in the late 
course of the disease following intraveneous gadolinium administration.

The diagnosis of spinal epidural abscess is rather easy when classical clinical, laboratory and imaging findings are present, or when it is accompanied by vertebral osteomyelitis. But in cases of spontaneous abscess formation, in the absence of predisposing factors or when clinical and laboratory findings are not suggestive of an abscess, like it was in our case, diagnosis may be a real challenge. Early diagnosis and treatment are of utmost importance in decreasing the mortality and morbidity risks of SEA considering its high mortality $(5-31 \%)$ and morbidity rates [8]. Cases of misdiagnosed spontaneous epidural abscesses showing significant contrast enhancement in the routine MR images have been reported in the medical literature [10]. In our case, however, the diagnosis of spontaneous epidural abscess was made by demonstrating the paraspinal inflammatory changes using fat-suppresses images in addition to the routine MRI sequences.

The most important prognostic factor in SEA cases is the neurological status of the patient at the time of diagnosis. Age above 60, location in the cervical region, extensive abscess, spinal cord symptoms lasting for more than $72 \mathrm{~h}$, presence of sepsis, low white blood cell count $(<100 \times 109 / 1)$ and extremely high ESR values $(>110 \mathrm{~mm} / \mathrm{h})$ are bad prognostic factors.

The standard therapy of spinal epidural abscess is surgical decompression, followed by intravenous antibiotic therapy for 2-6 weeks and another 4-6-week oral antibiotherapy [9].

In conclusion, because of the importance of its early diagnosis, posterior epidural abscess should be considered in the differential diagnosis of posterior epidural masses, especially in young individuals with no fever, no predisposing factors and normal laboratory values. Fat-suppressed MRI images aid routine MRI images in diagnosing spontaneous epidural abscesses by demonstrating the inflammatory changes in the paraspinal area.

\section{References}

1. Pereira CE, Lynch JC (2005) Spinal epidural abscess: an analysis of 24 cases. Surg Neurol 63:26-29

2. Chao D, Nanda A (2002) Spinal epidural abscess: a diagnostic challenge. Am Fam Phys 65:1341-1346

3. Klekamp J, Samii M (1999) Extradural infections of the spine. Spinal Cord 37:103-109

4. Tang HJ, Lin HJ, Liu YC, Li CM (2002) Spinal epidural abscessexperience with 46 patients and evaluation of prognostic factors. $\mathrm{J}$ Infect 45:76-81

5. Lu CH, Chang WN, Lui CC, Lee PY, Chang HW (2002) Adult spinal epidural abscess: clinical features and prognostic factors. Clin Neurol Neurosurg 104:306-310

6. Lasker BR, Harter DH (1987) Cervical epidural abscess. Neurology $37: 1747-1753$

7. Anand S, Maini L, Agarwal A, Singh T, Dhal AK, Dhaon BK (1999) Spinal epidural abscess - a report of six cases. Int Orthop 23:175-177

8. Rust TM, Kohan S, Steel T, Lonergan R (2005) CT guided aspiration of a cervical spinal epidural abscess. J Clin Neurosci 12:453-456

9. Murphy DR, Morris NJ (2006) Cervical epidural abscess in an afebrile patient: a case report. J Manip Physiol Ther 29:672675

10. Tang K, Xenos C, Sgouros S (2001) Spontaneous spinal epidural abscess in a neonate. With a review of the literature. Childs Nerv Syst 17:629-631 